\title{
Using integrated knowledge acquisition to prepare sophisticated expert plans for their re-use in novel situations
}

Franz Schmalhofer, Ralf Bergmann, Otto Kühn, Gabriele Schmidt

$$
\text { June } 1992
$$

Deutsches Forschungszentrum für Künstliche Intelligenz GmbH 


\section{Deutsches Forschungszentrum für Künstliche Intelligenz}

The German Research Center for Artificial Intelligence (Deutsches Forschungszentrum für Künstliche Intelligenz, DFKI) with sites in Kaiserslautern and Saarbrücken is a non-profit organization which was founded in 1988 by the shareholder companies Daimler-Benz, IBM, Insiders, Fraunhofer Gesellschaft, GMD, Krupp-Atlas, Digital-Kienzle, Philips, Sema Group Systems, Siemens and Siemens-Nixdorf. Research projects conducted at the DFKI are funded by the German Ministry for Research and Technology, by the shareholder companies, or by other industrial contracts.

The DFKI conducts application-oriented basic research in the field of artificial intelligence and other related subfields of computer science. The overall goal is to construct systems with technical knowledge and common sense which - by using Al methods - implement a problem solution for a selected application area. Currently, there are the following research areas at the DFKI:

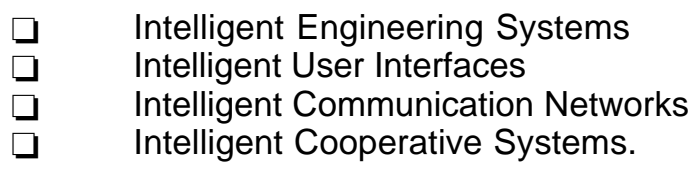

The DFKI strives at making its research results available to the scientific community. There exist many contacts to domestic and foreign research institutions, both in academy and industry. The DFKI hosts technology transfer workshops for shareholders and other interested groups in order to inform about the current state of research.

From its beginning, the DFKI has provided an attractive working environment for Al researchers from Germany and from all over the world. The goal is to have a staff of about 100 researchers at the end of the building-up phase.

Prof. Dr. Gerhard Barth

Director 
Using integrated knowledge acquisition to prepare sophisticated expert plans for their re-use in novel situations

Franz Schmalhofer, Ralf Bergmann, Otto Kühn, Gabriele Schmidt

DFKI-RR-92-25 
This paper has been published in the Conference Proceedings of the 15th German Workshop on Artificial Intelligence (GWAI-91).

This work has been supported by a grant from The Federal Ministry for Research and Technology (FKZ ITW-8902 C4).

This work may not be copied or reproduced in whole or in part for any commercial purpose. Permission to copy in whole or in part without payment of fee is granted for nonprofit educational and research purposes provided that all such whole or partial copies include the following: a notice that such copying is by permission of Deutsches Forschungszentrum für Künstliche Intelligenz, Kaiserslautern, Federal Republic of Germany; an acknowledgement of the authors and individual contributors to the work; all applicable portions of this copyright notice. Copying, reproducing, or republishing for any other purpose shall require a licence with payment of fee to Deutsches Forschungszentrum für Künstliche Intelligenz. 


\title{
Using Integrated Knowledge Acquisition to Prepare Sophisticated Expert Plans for Their Re-Use in Novel Situations
}

\author{
Franz Schmalhofer, Ralph Bergmann, Otto Kühn, Gabriele Schmidt \\ German Research Center for Artificial Intelligence \\ University Bldg 57 \\ Erwin-Schrödinger Str. \\ D-6750 Kaiserslautern \\ Germany \\ e-mail: schmalho@informatik.uni-kl.de
}

\begin{abstract}
Plans which were constructed by human experts and have been repeatedly executed to the complete satisfaction of some customer in a complex real world domain contain very valuable planning knowledge. In order to make this compiled knowledge re-usable for novel situations, a specific integrated knowledge acquisition method has been developed: First, a domain theory is established from documentation materials or texts, which is then used as the foundation for explaining how the plan achieves the planning goal. Secondly, hierarchically structured problem class definitions are obtained from the practitioners' highlevel problem conceptualizations. The descriptions of these problem classes also provide operationality criteria for the various levels in the hierarchy. A skeletal plan is then constructed for each problem class with an explanation-based learning procedure. These skeletal plans consist of a sequence of general plan elements, so that each plan element can be independently refined. The skeletal plan thus accounts for the interactions between the various concrete operations of the plan at a general level. The complexity of the planning problem is thereby factored in a domain-specific way and the compiled knowledge of sophisticated expert plans can be re-used in novel situations.
\end{abstract}

\section{MOTIVATION}

Like other synthetic tasks, planning problems are inherently intractable [Georgeff87]. In a complex real world domain such as production planning in mechanical engineering, expert systems can consequently not be based on planning from first principles [Koehler91]. It is also not surprising that in at least 80 percent of all mechanical engineering planning tasks, even human planners re-use old plans by adapting them to the new planning problem [Spur79; ThobenSchmalhofer90].

Expert plans have not only been developed with much effort, but were also carefully tested and have proven their sophistication during numerous successful executions in the real world. Preparing such human planning solutions for their re-use in novel situations can provide an important basis for the development of a successful planning system.

This paper describes a general procedure by which concrete human expert plans can be generalized into skeletal plans [FriedlandIwasaki85]. A skeletal plan provides a partitioning 
of the enormous search space of the complete planning problem into a number of subproblems with small search spaces. The skeletal plans constructed by this procedure are indexed by the various application conditions so that they can be re-used in novel situations.

Explanation-based learning [MitchellKeller86] is applied to find an appropriate generalization of a concrete case consisting of the description of a manufacturing problem and its solution. It is embedded into an integrated knowledge acquisition method [SchmalhoferKuehn+91] which provides the domain theory and allows the specification of domain-adequate operationality criteria for the construction of skeletal plans.

We will first outline the integrated knowledge acquisition framework, which is based on a quite general model of expertise. The general model describes the overall structure of the future expert system. We will then describe the skeletal plan construction procedure and its implementation in some detail. The application of the procedure to the production planning of a rotational part will be described and the results will be discussed.

\section{INTEGRATED KNOWLEDGE ACQUISITION FOR PLAN RE-USE}

The problem of production planning in mechanical engineering consists of finding an adequate production plan for a given workpiece which is to be manufactured in some factory. For the manufacturing of a rotational part, the production plan consists of a sequence of chucking and cutting operations by which the workpiece can be manufactured.

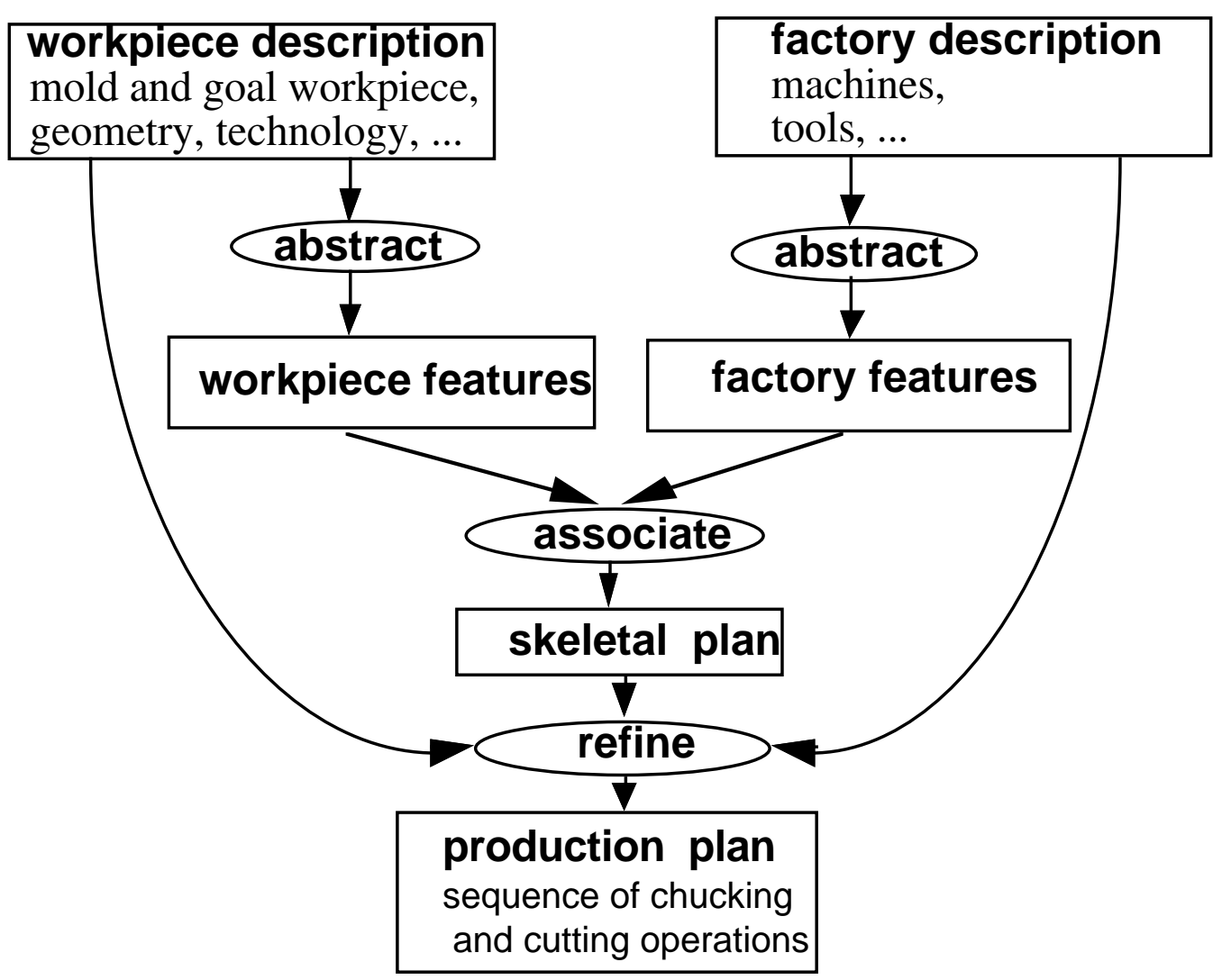

Figure 1: Model of expertise for production planning 
The general structure of the expert system which is being developed can be described by the model of expertise [BreukerWielinga89] shown in Figure 1. From the concrete description of the workpiece and the available manufacturing environment more abstract feature descriptions are first constructed. These abstractions are then associated with an appropriate skeletal plan that has been stored stored in the knowledge base. The skeletal plan is finally refined with the help of the workpiece and the factory description into the concrete production plan.

The model of expertise specifies what kind of knowledge has to be acquired for the expert system, namely abstraction rules, refinement rules and skeletal plans which are associated with features of the problem description. In addition, a model of mechanical engineering actions is presumed as a general domain model. This model requires chucking and cutting operations to be described by some typology and their preconditions and effects.

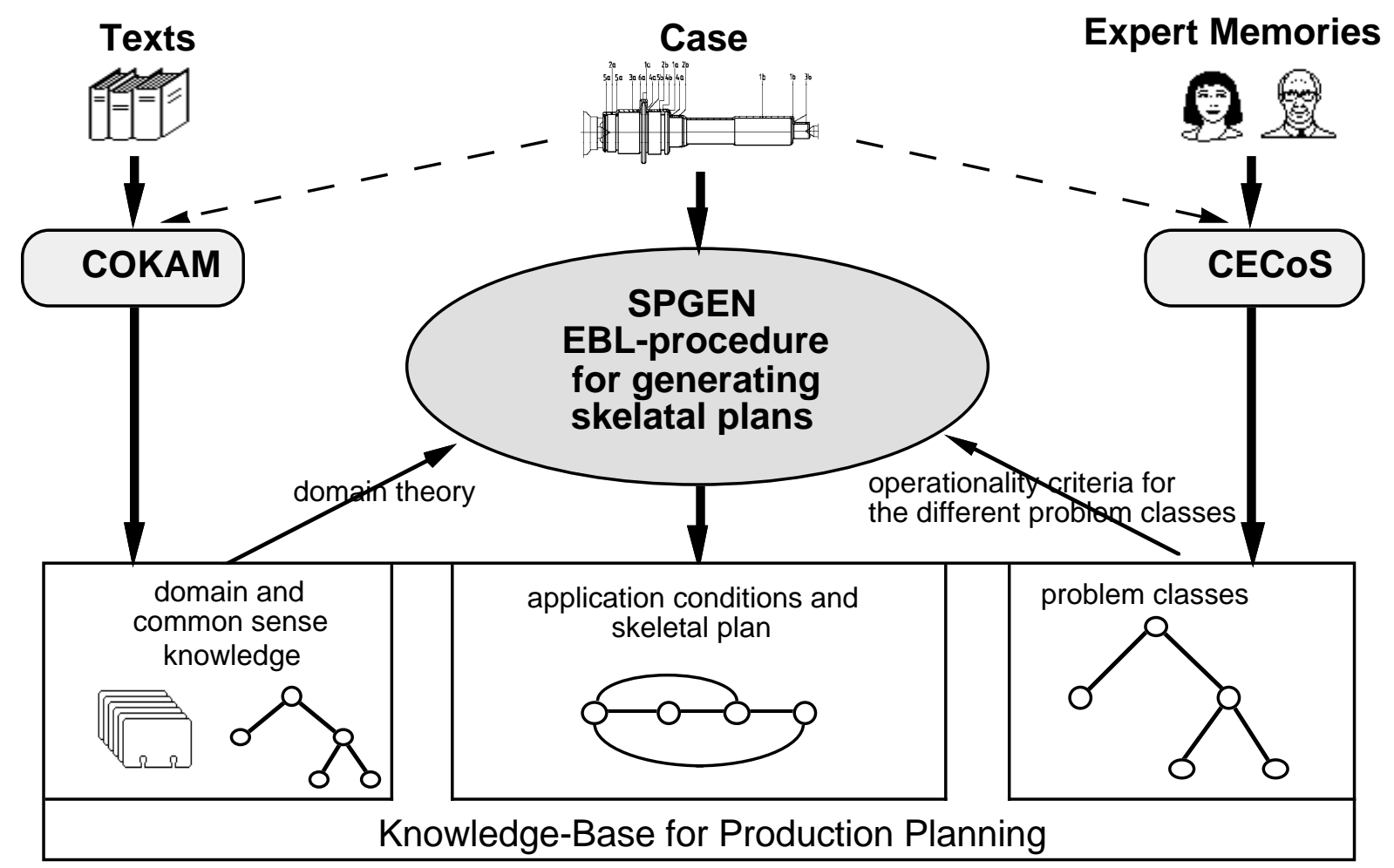

Figure 2: Integrated knowledge acquisition method

An integrated knowledge acquisition method is used to coordinate knowledge from texts, previously solved planning problems (cases) and the expert's respective memories. The knowledge acquisition tools COKAM (Case-Oriented Knowledge-Acquisition Method from Text) [SchmidtSchmalhofer90; KuehnLinster+91] and CECoS (C ase-Experience Combination System) [BergmannSchmalhofer91] are applied to the same set of cases so that the knowledge acquired with the two tools will complement one another. The domain and common sense knowledge supplied by COKAM and the definition of production classes 
obtained through CECoS, can then be utilized to automatically construct skeletal plans and associated application conditions through the explanation-based learning procedure SPGEN (Skeletal Plan Generation Procedure).

\subsection{Case oriented knowledge acquisition with COKAM}

With the interactive tool COKAM information is interactively extracted from a text and subsequently enhanced by the expert's elaborations. The extracted information is then mapped to the model of mechanical engineering actions (domain model). The so collected knowledge thus provides an explanation of each step in the production plan and specifies the conditions which are required for its application and the resulting consequences. Table 1 shows a sample of text information and expert elaborations, which are relevant for determining the preconditions and consequences of a specific cutting operation. The mapping of the 3rd knowledge unit of Table 1 into the model of mechanical engineering actions, which will be described in section 3.1 shows that the extracted information needs to be properly interpreted.

1. For rough cutting the cutting speed should be 400 to $600 \mathrm{~m} /$ minute.

2. When the mold has been forged, bezeling is required, if ceramic cutting tools are to be used.

3. The surface roughness Rt depends on the cutting feed $f$ and the corner radius $r_{e}$ of the cutting tool and can be computed by the formula $R_{t}=$ $\mathrm{f}^{2} / 8 \mathrm{re}$.

4. When thin workpieces are manufactured with a high cutting force, vibrations may occur.

5. When high tolerances are required, a very hard cutting material must be used for fine turning.

Table 1: A sample of text information extracted with COCAM

\subsection{Acquisition of problem classes with CECoS}

With the interactive tool CECoS a hierarchically structured set of problem classes is obtained from a set of prototypical cases and human expert judgements. The problem classes are defined so that a useful skeletal plan will exist for each problem class. From explicit and implicit memories, the expert first establishes an extensional definition of the various problem classes with respect to selected prototypical cases. The so established production classes are then intensionally and thereby generally defined.

Because the class definitions are based on expert judgements, the classes should be defined at the right level of generality: They should be general enough so that a large number of specific problems fall into the different classes and they should be specific enough to 
provide operational knowledge for production planning.

Figure 3 shows a section of the hierarchy of production classes which was obtained for some prototypical shafts. Class $\mathrm{A}$ is defined by the features which all three cases have in common. The more specific class B inherits all the features from class A and has some additional features which apply to the cases M5 and M4 but not to M3.

The features of each class may refer to the geometry (long workpiece) and technology (hardened steel) of the workpiece, to the factory (one tool revolver), or to the production plan ( 2 chucking fixations). As will be shown later, the features referring to the problem description (i.e. the workpiece or the factory) are utilized for the specification of the application conditions, whereas the features referring to the production plan are used for the definition of the operator classes in the skeletal plans.

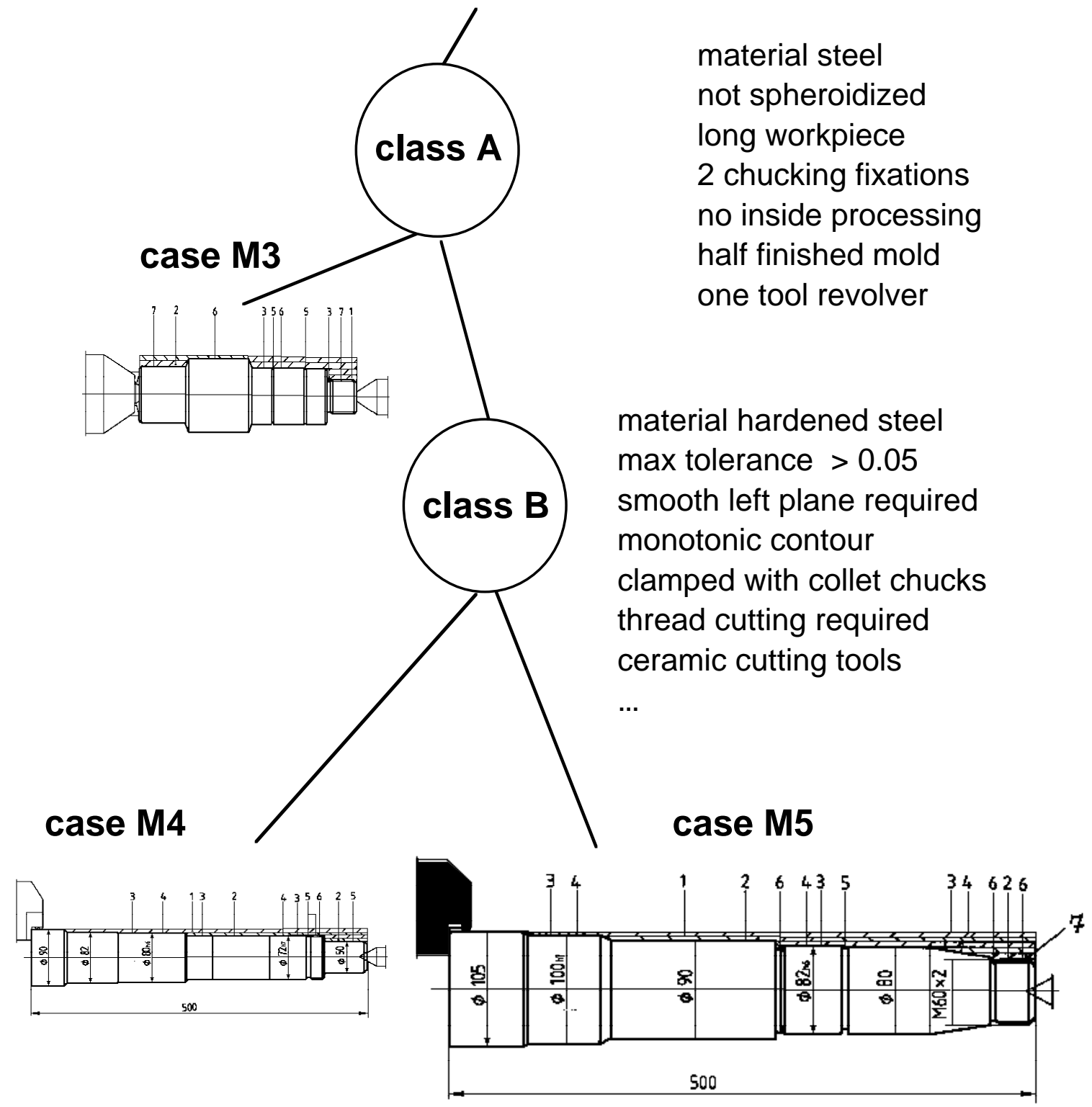

Figure 3: A section of a hierarchy of problem classes acquired with CECoS 


\section{PROCEDURE FOR GENERATING SKELETAL PLANS}

SPGEN is based on explanation-based generalization as described by [MitchellKeller86]. The domain and common sense knowledge acquired with COKAM is thereby used as domain theory and the hierarchy of problem classes is employed to specify operationality criteria. Depending upon the selected problem class and the respective operationality criteria, a more or less general skeletal plan will be obtained from a given case.

A skeletal plan is constructed by SPGEN in four phases:

1. In the first phase the execution of the source plan is simulated and explanations for the effects of the individual operations are constructed.

2. In the second phase the generalization of these explanations is performed with respect to a criterion of operationality, that specifies the vocabulary for defining abstract operators for the skeletal plan.

3. In the third phase, a dependency analysis of the resulting operator effects unveils the substantial interactions of the concrete plan at the more general level of the skeletal plan.

4. In the forth phase the concept descriptions for the abstract operators of the skeletal plan are formed by collecting and normalizing the important constraints for each operation that were indicated by the dependencies.

For describing the SPGEN procedure we will use a simplification of the case M5 from Figure 3. The input and the (intermediate) results of the procedure will be presented in a PROLOGlike notation in which unquoted strings beginning with an upper-case character denote variables.

The formal representation of the case M5 which is used as input to SPGEN is shown in Table 2. The left side of the table shows the representation of the problem description which consists of the representation of the to be manufactured workpiece, the mold and the factory. The geometry of the workpiece and the geometry of the mold is represented by elementary surfaces. The technology is represented by a specification of the tolerances, the material, the heat-treatment, etc. These specifications may apply to individual or to all surfaces. The production plan is represented as a sequence of chucking and cutting operations with various parameters. 


\begin{tabular}{|c|c|}
\hline 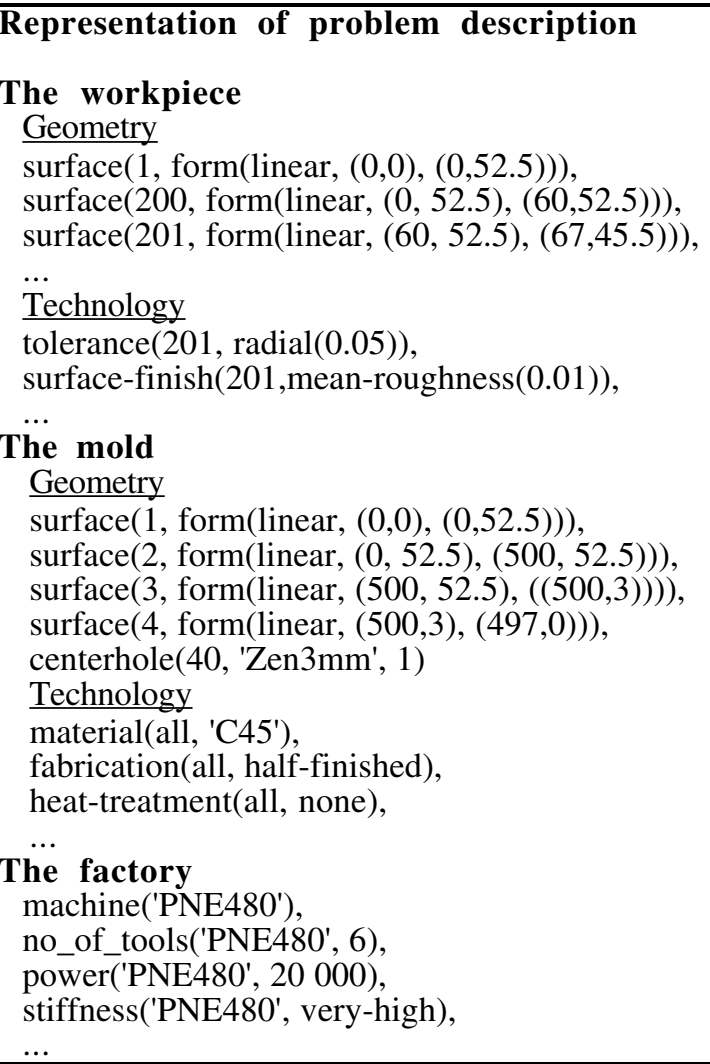 & 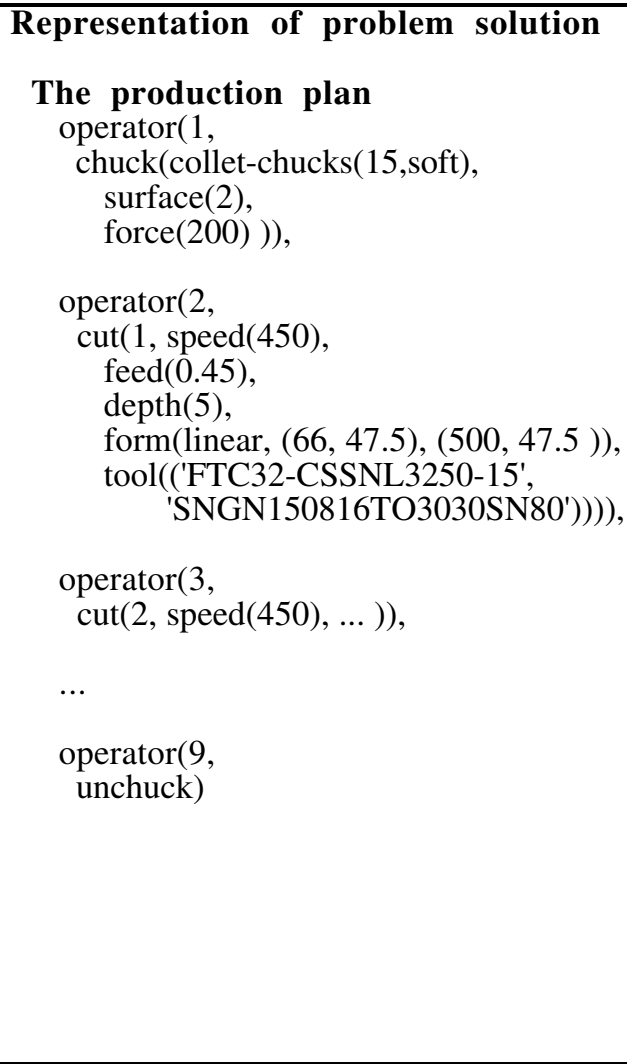 \\
\hline
\end{tabular}

Table 2: Partial representation of a case used as input for SPGEN

\subsection{Simulation and Explanation}

In the first phase of SPGEN, the plan execution is simulated on the basis of the available domain theory. The simulation of the plan is performed by sequentially determining the effects of each operator $\mathrm{Op}_{1}, \ldots, \mathrm{Op}_{\mathrm{n}}$ of the plan. In order to determine the effects of the sequence of operators, the intermediate processing states from the initial state $\mathrm{S}_{0}$ (the mold) to the final state $S_{n}$ (which will contain the target workpiece if the domain theory is sufficient) are computed as follows:

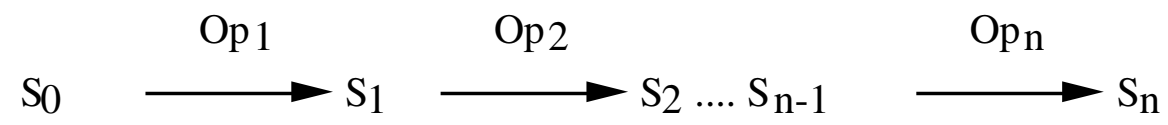

The effects of the operator are represented by a set of rules with STRIPS like add- and delete actions. The execution of these rules thus create the successor world state. For example, knowledge unit 3 from table 1 is represented by the following rule:

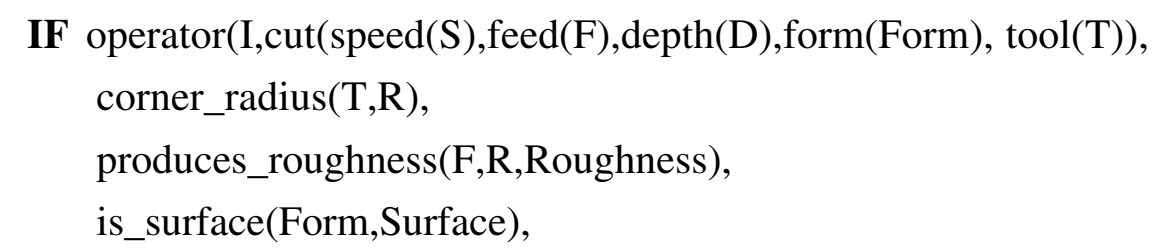

THEN ADD(roughness(Surface,Roughness))

By applying all the rules for each operator, the various consequences of the individual operations of the plan are calculated. If the domain theory is sufficient, a complete 
explanation of the plan will be obtained. The proofs that exist for the applicability of each operator rule can now be seen as an explanation of each effect that depends on operator attributes as well as world state attributes, from the initial or intermediate states.

\subsection{Generalization}

In the second phase of the procedure, these proofs are independently generalized for each production step of the plan (explanation based generalization). The independent generalization of each production step is necessitated because of the complexity of the complete plans.

The degree of generalization is determined by the operationality criteria for each production step, which are defined at the concept [Hirsh88] rather than at the predicate level. These criteria are obtained from the terms, which the texts and the expert used for describing the different operations of the concrete plan at a general level. It is thus assumed that exactly those terms which are used by experienced humans would determine operationality. A justification for this assumption can be found in the research of Rosch [Rosch78]: Rosch has shown that humans favor basic level categories in their descriptions. Such categories can be termed operational in the sense that they provide maximum information and the least cognitive effort for achieving some task goal.

\subsection{Dependency Analysis}

The dependency analysis of the third phase determines which previous operations (or initial state affairs) achieved the prerequisites for the various productions steps of the plan. It is thereby determined when the prerequisites for performing a specific production step were accomplished. A directed graph is constructed, in which all existing dependencies between the individual plan operations and the problem description are denoted by arcs. These problem descriptions, which were obtained through CECoS determine the generality of the skeletal plan to be constructed. The operationality criteria are provided by the features of the problem classes which were acquired from the human expert with the knowledge acquisition tool CECoS.

With the hierarchy of problem classes shown in figure 3, either the features of class B (and its subclasses) or the features of class A (and its subclasses) can be specified as being operational. In the first case a rather specific skeletal plan which applies to the problems of class B will be constructed, whereas in the latter case a more general skeletal plan for class A will be obtained.

Figure 4 shows a graphical representation of a part of the dependency graph that results from the analysis of the case M5. For example, cut 1 depends on the workpiece being chucked (for subsequent cuts this obvious dependency is no longer shown in the graph), on the geometry and the technology of the mold, and on the availability of ceramic cutting tools in the factory. It can also be seen from figure 4 that the first three cuts produce intermediate surfaces which are needed for the subsequent cut respectively but are no longer present in the 
final workpiece. The required geometry and technology of the goal workpiece is produced exclusively by the cuts 4 to 7 , each of which produces some particular feature. The lack of a dependency between the cuts 5 to 7 , furthermore indicates that they could be executed in any sequence.

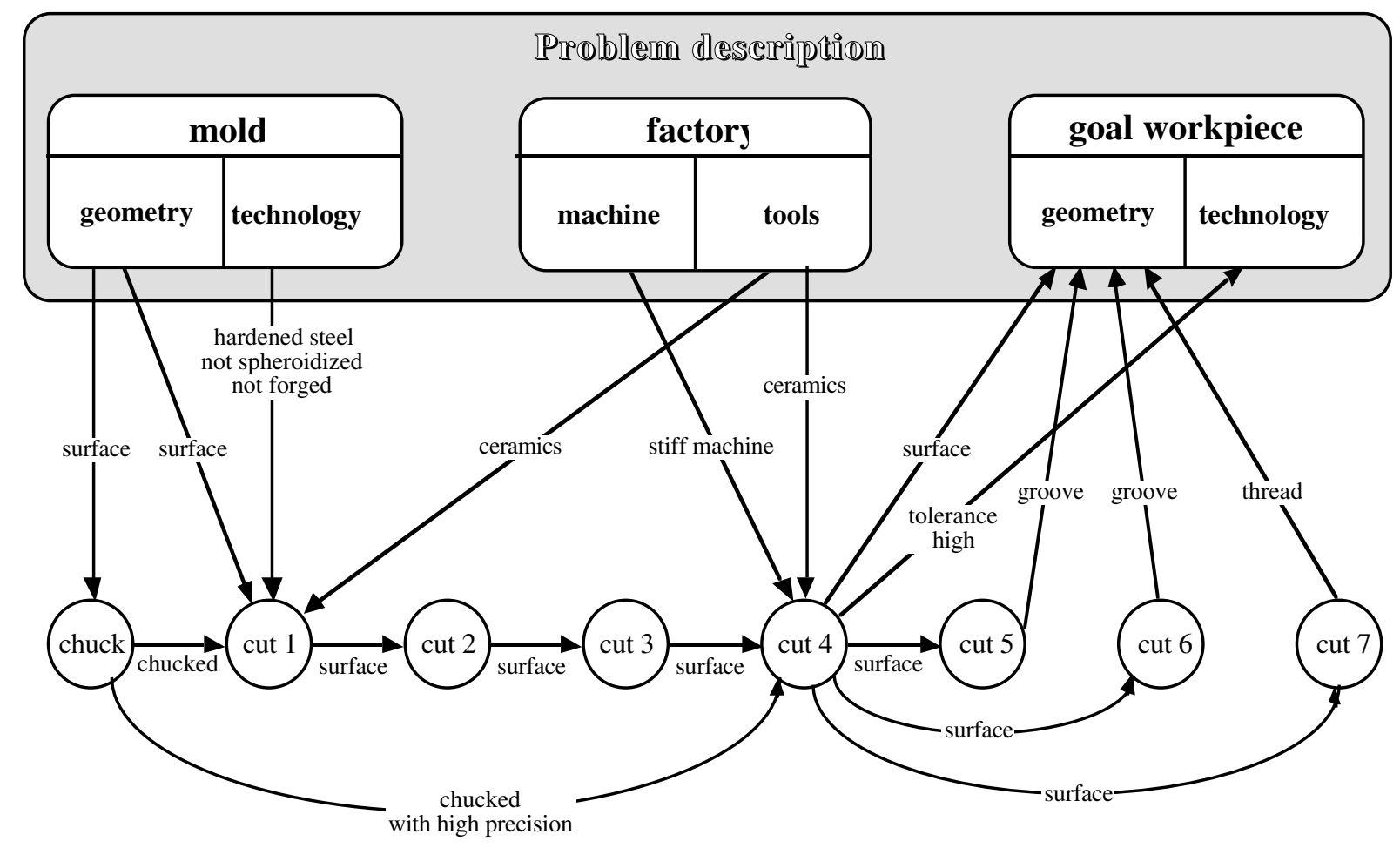

Figure 4: Partial dependency graph for the case M5

\subsection{Normalization}

This last phase builds the skeletal plan in its final representation by identifying independently solvable sub-formulas from the dependency graph which expresses only local constraints on one operator. By analyzing the occurrence of variables in the graph the dependencies are separated into:

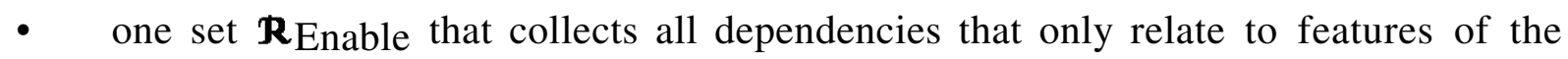
problem description,

- $\quad$ one set $\boldsymbol{R}_{\text {Opi }}$ for each operator Opi where the dependencies refer to parameters of the operator Opi

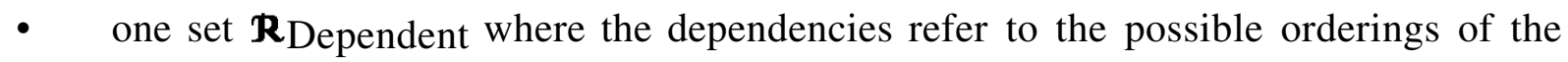
operator classes.

The set of constraints $\mathbf{R}_{\text {Enable formally describes the class of problems for which the skeletal }}$ plan can be used: it specifies the application conditions for the skeletal plan. The application 
conditions may refer to the mold, the goal workpiece or the manufacturing environment, as indicated in Figure 4. The skeletal plan itself consists of the set of operator classes Op $1, \ldots, \mathrm{Op}_{\mathrm{n}}$ with the constraints $\boldsymbol{R}_{\mathrm{Opi}}$ and $\boldsymbol{R}_{\text {Dependent which specify the possible }}$ sequences in which they may be applied.

For the case M5 the skeletal plan with application conditions shown in Table 3 is generated for the problem class B. The skeletal plan for the problem class A would be somewhat more general. For instance, it would allow any chucking tool with two fixations instead of collet chucks and would not require the material to be hardened steel.

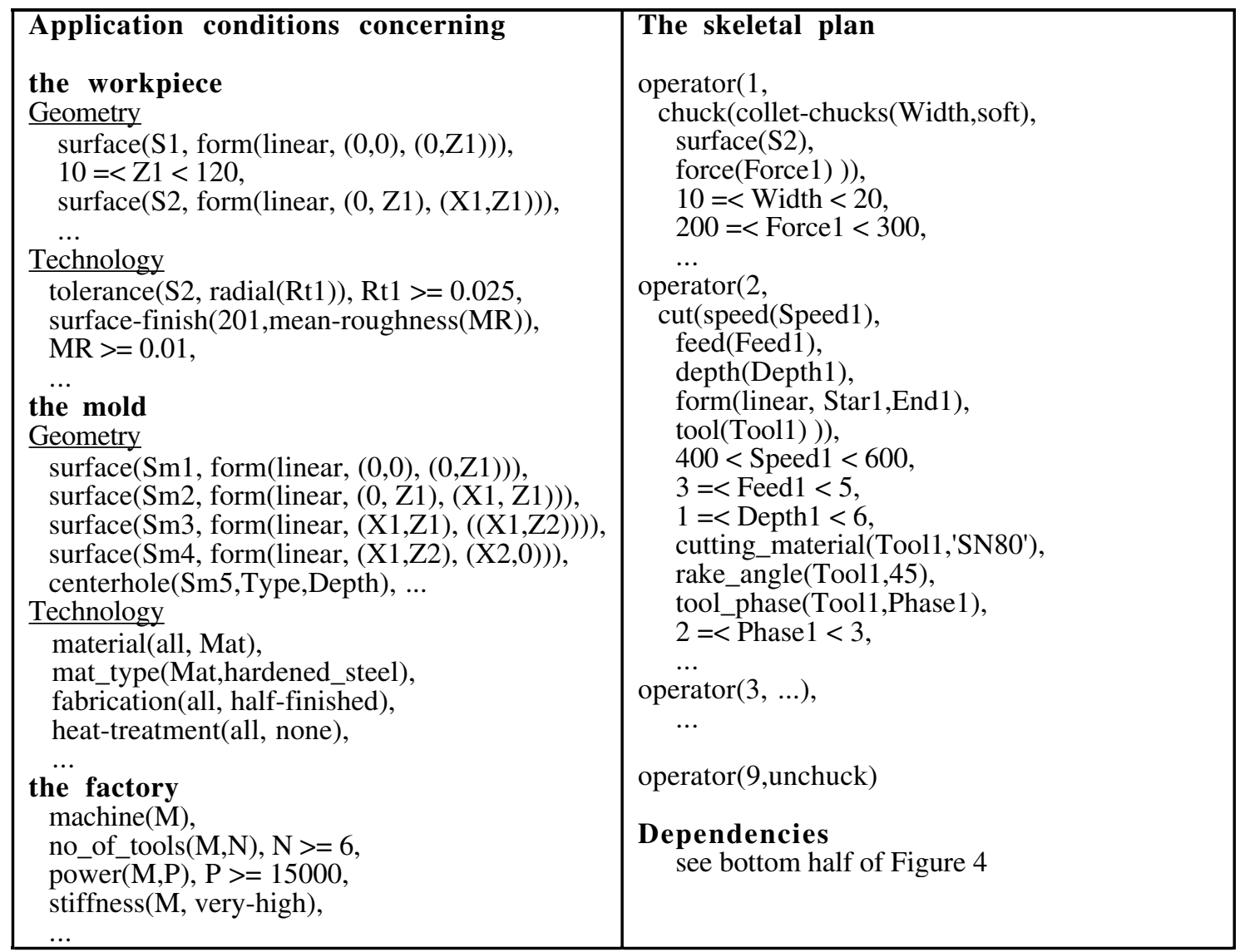

Table 3: Partial skeletal plan generated from case M5 for problem class A

A first version of SPGEN has been implemented in LPA-PROLOG on a MAC II computer [Bergmann90]. It can construct skeletal plans from simplified cases such as those shown in Figure 3. The current implementation deals mostly with the geometrical aspects and does not yet adequately take into account the technological and economical aspects of production planning. 


\section{DISCUSSION}

The re-use of previously established solutions to hard problems has been suggested in the area of Artificial Intelligence [RiesbeckSchank89] as well as for software development in general [Fischer87; Standish84]. In the area of Artificial Intelligence most approaches to the re-use of established solutions are discussed within the framework of case-based reasoning [Koehler91]. In case-based reasoning, the modification of an old case to a new problem is typically performed at the time when the new problem arises. By suggesting to systematically prepare sophisticated expert plans already during the knowledge acquisition process for an expert system these approaches are extended in the current paper.

Unlike case-based planning, the preparation of a case for its re-use is thus performed in ignorance of a specific new problem. It basically consists in analyzing and explaining a prototypical case in terms of a model of expertise and supplementary domain knowledge. Additionally, the features of problem classes which supposedly constitute the base level categories of human experts [Rosch78] are used to determine operationality criteria for concepts in an explanation-based generalization procedure.

The skeletal plans and application conditions constructed with SPGEN, provide a combination of knowledge-based and heuristic abstractions of a concrete plan. For novel problems, which satisfy the application conditions, the skeletal plan will provide a knowledgebased partitioning of the novel problems into appropriate subproblems, which can then be solved more easily.

\section{REFERENCES}

[Bergmann90] Bergmann, R. (1990). Generierung von Skelettplänen als Problem der

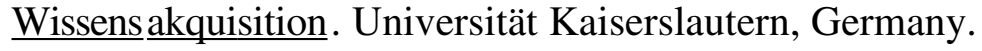

[BergmannSchmalhofer91] Bergmann, R., \& Schmalhofer, F. (1991). CECoS: A case experience combination system for knowledge acquisition for expert systems. Behavior Research Methods, Instruments, \& Computers. in press.

[BreukerWielinga89] Breuker, J., \& Wielinga, B. (1989). Models of expertise in knowledge acquisition. In Guida, G., \& Tasso, C. (Eds.), Topics in expert system design, methodologies and tools (pp. 265 - 295). Amsterdam: North Holland.

[Fischer87] Fischer, G. (1987, July). Cognitive view of reuse and redesign. IEEE Software, 60-72.

[FriedlandIwasaki85] Friedland, P.E., \& Iwasaki, Y. (1985). The concept and implementation of skeletal plans. Journal of Automated Reasoning, 161-208.

[Georgeff87] Georgeff, M.P. (1987). Planning. Annual Reviews in Computing Science, (2), 359-400.

[Hirsh88] Hirsh, H. (1988). Reasoning about operationality for explanation-based learning. Proceedings of the 5th International Conference on Machine Learning, 214 - 220. 
[Koehler91] Köhler, J. (1991). Approaches to the reuse of plan schemata in planning formalisms (Technical Memo No. TN-91-01). Kaiserslautern, Germany: German Research Center for Artificial Intelligence.

[KuehnLinster+91] Kühn, O., Linster, M., \& Schmidt, G. (1991, May). Clamping, COKAM, KADS, and OMOS: The construction and operationalization of a KADS conceptual model. Proceedings of EKAW 91 (Crieff) .

[MitchellKeller86] Mitchell, T.M., Keller, R.M., \& Kedar-Cabelli, S.T. (1986). Explanation-based generalization: A unifying view. Machine Learning, (1), 47 - 80.

[RiesbeckSchank89] Riesbeck, C.K., \& Schank, R.C. (1989). Inside case-based reasoning. Hillsdale Lawrence Earlbaum.

[Rosch78] Rosch, E. (1978). Principles of categorisation. In Rosch, E., \& Lloyd, B. (Eds.), Cognition and categorisation. Hillsdale, New Jersey: Lawrence Erlbaum.

[SchmalhoferKuehn+91] Schmalhofer, F., Kühn, O., \& Schmidt, G. (in press). Integrated knowledge acquisition from text, previously solved cases, and expert memories . Applied Artificial Intelligence.

[SchmidtSchmalhofer90] Schmidt, G., \& Schmalhofer, F. (1990). Case-oriented knowledge acquisition from texts. In Wielinga, B., Boose, J., Gaines, B., Schreiber, G., \& van Someren, M. (Eds.), Current trends in knowledge acquisition (pp. 302312). Amsterdam: IOS Press.

[Spur79] Spur, G. (1979). Produktionstechnik im Wandel. München: Carl Hanser Verlag.

[Standish84] Standish, T.S. (1984). An essay on software reuse. IEEE Transactions on Software Engineering, 10 (5), 494 - 497.

[ThobenSchmalhofer90] Thoben, J., \& Schmalhofer, F. (1990). WiederholungsVarianten- und Neuplanung bei der Fertigung rotationssymmetrischer Teile (Interner Bericht des ARC-TEC-Projektes). Kaiserslautern, Germany: German Research Center for Artificial Intelligence. 


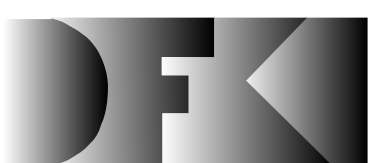

Deutsches

Forschungszentrum

für Künstliche

Intelligenz GmbH
DFKI

-Bibliothek-

PF 2080

67608 Kaiserslautern

FRG

\section{DFKI Publikationen}

Die folgenden DFKI Veröffentlichungen sowie die aktuelle Liste von allen bisher erschienenen Publikationen können von der oben angegebenen Adresse oder per anonymem ftp von ftp.dfki.unikl.de (131.246.241.100) unter pub/Publications bezogen werden.

Die Berichte werden, wenn nicht anders gekennzeichnet, kostenlos abgegeben.

\section{DFKI Research Reports}

RR-92-45

Elisabeth André, Thomas Rist: The Design of Illustrated Documents as a Planning Task 21 pages

\section{RR-92-46}

Elisabeth André, Wolfgang Finkler, Winfried Graf, Thomas Rist, Anne Schauder, Wolfgang Wahlster: WIP: The Automatic Synthesis of Multimodal Presentations

19 pages

\section{RR-92-47}

Frank Bomarius: A Multi-Agent Approach towards Modeling Urban Traffic Scenarios

24 pages

RR-92-48

Bernhard Nebel, Jana Koehler:

Plan Modifications versus Plan Generation:

A Complexity-Theoretic Perspective

15 pages

RR-92-49

Christoph Klauck, Ralf Legleitner, Ansgar Bernardi: Heuristic Classification for Automated CAPP 15 pages

\section{RR-92-50}

Stephan Busemann:

Generierung natürlicher Sprache

61 Seiten

\section{RR-92-51}

Hans-Jürgen Bürckert, Werner Nutt:

On Abduction and Answer Generation through Constrained Resolution

20 pages

\section{RR-92-52}

Mathias Bauer, Susanne Biundo, Dietmar Dengler, Jana Koehler, Gabriele Paul: PHI - A Logic-Based Tool for Intelligent Help Systems

14 pages

\section{DFKI Publications}

The following DFKI publications or the list of all published papers so far are obtainable from the above address or per anonymous ftp from ftp.dfki.uni-kl.de (131.246.241.100) under pub/Publications.

The reports are distributed free of charge except if otherwise indicated.

\section{RR-92-53}

Werner Stephan, Susanne Biundo:

A New Logical Framework for Deductive Planning 15 pages

\section{RR-92-54}

Harold Boley: A Direkt Semantic Characterization of RELFUN

30 pages

\section{RR-92-55}

John Nerbonne, Joachim Laubsch, Abdel Kader

Diagne, Stephan Oepen: Natural Language

Semantics and Compiler Technology

17 pages

\section{RR-92-56}

Armin Laux: Integrating a Modal Logic of Knowledge into Terminological Logics 34 pages

\section{RR-92-58}

Franz Baader, Bernhard Hollunder:

How to Prefer More Specific Defaults in

Terminological Default Logic

31 pages

\section{RR-92-59}

Karl Schlechta and David Makinson: On Principles and Problems of Defeasible Inheritance 13 pages

RR-92-60

Karl Schlechta: Defaults, Preorder Semantics and Circumscription

19 pages

RR-93-02

Wolfgang Wahlster, Elisabeth André, Wolfgang Finkler, Hans-Jürgen Profitlich, Thomas Rist: Plan-based Integration of Natural Language and Graphics Generation 50 pages 


\section{RR-93-03}

Franz Baader, Berhard Hollunder, Bernhard Nebel, Hans-Jürgen Profitlich, Enrico Franconi: An Empirical Analysis of Optimization Techniques for Terminological Representation Systems 28 pages

\section{RR-93-04}

Christoph Klauck, Johannes Schwagereit:

GGD: Graph Grammar Developer for features in CAD/CAM

13 pages

\section{RR-93-05}

Franz Baader, Klaus Schulz: Combination Techniques and Decision Problems for Disunification 29 pages

\section{RR-93-06}

Hans-Jürgen Bürckert, Bernhard Hollunder, Armin Laux: On Skolemization in Constrained Logics 40 pages

RR-93-07

Hans-Jürgen Bürckert, Bernhard Hollunder, Armin Laux: Concept Logics with Function Symbols 36 pages

\section{RR-93-08}

Harold Boley, Philipp Hanschke, Knut Hinkelmann, Manfred Meyer: COLAB: A Hybrid Knowledge Representation and Compilation Laboratory 64 pages

\section{RR-93-09}

Philipp Hanschke, Jörg Würtz:

Satisfiability of the Smallest Binary Program 8 Seiten

\section{RR-93-10}

Martin Buchheit, Francesco M. Donini, Andrea Schaerf: Decidable Reasoning in Terminological Knowledge Representation Systems

35 pages

\section{RR-93-11}

Bernhard Nebel, Hans-Juergen Buerckert:

Reasoning about Temporal Relations:

A Maximal Tractable Subclass of Allen's Interval Algebra

28 pages

\section{RR-93-12}

Pierre Sablayrolles: A Two-Level Semantics for French Expressions of Motion 51 pages

\section{RR-93-13}

Franz Baader, Karl Schlechta:

A Semantics for Open Normal Defaults via a

Modified Preferential Approach

25 pages

\section{RR-93-14}

Joachim Niehren, Andreas Podelski,Ralf Treinen: Equational and Membership Constraints for Infinite Trees

33 pages

\section{RR-93-15}

Frank Berger, Thomas Fehrle, Kristof Klöckner, Volker Schölles, Markus A. Thies, Wolfgang Wahlster: PLUS - Plan-based User Support

Final Project Report

33 pages

\section{RR-93-16}

Gert Smolka, Martin Henz, Jörg Würtz: ObjectOriented Concurrent Constraint Programming in $\mathrm{Oz}$

17 pages

\section{RR-93-17}

Rolf Backofen:

Regular Path Expressions in Feature Logic 37 pages

\section{RR-93-18}

Klaus Schild: Terminological Cycles and the Propositional $\mu$-Calculus

32 pages

\section{RR-93-20}

Franz Baader, Bernhard Hollunder: Embedding Defaults into Terminological Knowledge Representation Formalisms 34 pages

\section{RR-93-22}

Manfred Meyer, Jörg Müller:

Weak Looking-Ahead and its Application in Computer-Aided Process Planning 17 pages

RR-93-23

Andreas Dengel, Ottmar Lutzy: Comparative Study of Connectionist Simulators 20 pages

\section{RR-93-24}

Rainer Hoch, Andreas Dengel:

Document Highlighting -

Message Classification in Printed Business Letters 17 pages

\section{RR-93-25}

Klaus Fischer, Norbert Kuhn: A DAI Approach to Modeling the Transportation Domain 93 pages

\section{RR-93-26}

Jörg P. Müller, Markus Pischel: The Agent Architecture InteRRaP: Concept and Application 99 pages

\section{RR-93-27}

Hans-Ulrich Krieger:

Derivation Without Lexical Rules 33 pages

RR-93-28

Hans-Ulrich Krieger, John Nerbonne, Hannes Pirker: Feature-Based Allomorphy 8 pages

\section{RR-93-29}

Armin Laux: Representing Belief in Multi-Agent Worlds viaTerminological Logics 35 pages 
RR-93-33

Bernhard Nebel, Jana Koehler:

Plan Reuse versus Plan Generation: A Theoretical and Empirical Analysis

33 pages

RR-93-34

Wolfgang Wahlster:

Verbmobil Translation of Face-To-Face Dialogs 10 pages

\section{RR-93-35}

Harold Boley, François Bry, Ulrich Geske (Eds.): Neuere Entwicklungen der deklarativen KIProgrammierung - Proceedings

150 Seiten

Note: This document is available only for a nominal charge of $25 \mathrm{DM}$ (or $15 \mathrm{US}-\$$ ).

\section{RR-93-36}

Michael M. Richter, Bernd Bachmann, Ansgar Bernardi, Christoph Klauck, Ralf Legleitner, Gabriele Schmidt: Von IDA bis IMCOD:

Expertensysteme im CIM-Umfeld 13 Seiten

\section{RR-93-38}

Stephan Baumann: Document Recognition of Printed Scores and Transformation into MIDI 24 pages

\section{RR-93-40}

Francesco M. Donini, Maurizio Lenzerini, Daniele Nardi, Werner Nutt, Andrea Schaerf:

Queries, Rules and Definitions as Epistemic Statements in Concept Languages

23 pages

\section{RR-93-41}

Winfried H. Graf: LAYLAB: A Constraint-Based Layout Manager for Multimedia Presentations 9 pages

\section{RR-93-42}

Hubert Comon, Ralf Treinen:

The First-Order Theory of Lexicographic Path Orderings is Undecidable

9 pages

\section{RR-93-44}

Martin Buchheit, Manfred A. Jeusfeld, Werner Nutt, Martin Staudt: Subsumption between Queries to Object-Oriented Databases 36 pages

\section{RR-93-45}

Rainer Hoch: On Virtual Partitioning of Large Dictionaries for Contextual Post-Processing to Improve Character Recognition 21 pages

\section{RR-93-46}

Philipp Hanschke: A Declarative Integration of Terminological, Constraint-based, Data-driven, and Goal-directed Reasoning 81 pages

\section{DFKI Technical Memos}

\section{TM-91-15}

Stefan Busemann: Prototypical Concept Formation An Alternative Approach to Knowledge Representation 28 pages

\section{TM-92-01}

Lijuan Zhang: Entwurf und Implementierung eines Compilers zur Transformation von Werkstückrepräsentationen

34 Seiten

\section{TM-92-02}

Achim Schupeta: Organizing Communication and Introspection in a Multi-Agent Blocksworld 32 pages

\section{TM-92-03}

Mona Singh:

A Cognitiv Analysis of Event Structure

21 pages

\section{TM-92-04}

Jürgen Müller, Jörg Müller, Markus Pischel, Ralf Scheidhauer:

On the Representation of Temporal Knowledge 61 pages

\section{TM-92-05}

Franz Schmalhofer, Christoph Globig, Jörg Thoben: The refitting of plans by a human expert 10 pages

TM-92-06

Otto Kühn, Franz Schmalhofer: Hierarchical skeletal plan refinement: Task- and inference structures

14 pages

\section{TM-92-08}

Anne Kilger: Realization of Tree Adjoining Grammars with Unification

27 pages

\section{TM-93-01}

Otto Kühn, Andreas Birk: Reconstructive Integrated Explanation of Lathe Production Plans 20 pages

\section{TM-93-02}

Pierre Sablayrolles, Achim Schupeta:

Conlfict Resolving Negotiation for COoperative Schedule Management 21 pages

\section{TM-93-03}

Harold Boley, Ulrich Buhrmann, Christof Kremer: Konzeption einer deklarativen Wissensbasis über recyclingrelevante Materialien 11 pages

\section{TM-93-04}

Hans-Günther Hein: Propagation Techniques in WAM-based Architectures — The FIDO-III Approach 105 pages 


\section{DFKI Documents}

\section{D-92-23}

Michael Herfert: Parsen und Generieren der

Prolog-artigen Syntax von RELFUN

51 Seiten

\section{D-92-24}

Jürgen Müller, Donald Steiner (Hrsg.):

Kooperierende Agenten

78 Seiten

\section{D-92-25}

Martin Buchheit: Klassische Kommunikations- und Koordinationsmodelle

31 Seiten

\section{D-92-26}

Enno Tolzmann:

Realisierung eines Werkzeugauswahlmoduls mit Hilfe des Constraint-Systems CONTAX

28 Seiten

\section{D-92-27}

Martin Harm, Knut Hinkelmann, Thomas Labisch: Integrating Top-down and Bottom-up Reasoning in COLAB

40 pages

\section{D-92-28}

Klaus-Peter Gores, Rainer Bleisinger: Ein Modell zur Repräsentation von Nachrichtentypen 56 Seiten

\section{D-93-01}

Philipp Hanschke, Thom Frühwirth: Terminological Reasoning with Constraint Handling Rules 12 pages

D-93-02

Gabriele Schmidt, Frank Peters,

Gernod Laufkötter: User Manual of COKAM+ 23 pages

\section{D-93-03}

Stephan Busemann, Karin Harbusch(Eds.): DFKI Workshop on Natural Language Systems: Reusability and Modularity - Proceedings 74 pages

\section{D-93-04}

DFKI Wissenschaftlich-Technischer Jahresbericht 1992

194 Seiten

\section{D-93-05}

Elisabeth André, Winfried Graf, Jochen Heinsohn, Bernhard Nebel, Hans-Jürgen Profitlich, Thomas Rist, Wolfgang Wahlster:

PPP: Personalized Plan-Based Presenter 70 pages

\section{D-93-06}

Jürgen Müller (Hrsg.):

Beiträge zum Gründungsworkshop der Fachgruppe Verteilte Künstliche Intelligenz Saarbrücken 29.30. April 1993

235 Seiten

Note: This document is available only for a nominal charge of $25 \mathrm{DM}$ (or 15 US-\$).

\section{D-93-07}

Klaus-Peter Gores, Rainer Bleisinger:

Ein erwartungsgesteuerter Koordinator zur partiellen Textanalyse

53 Seiten

\section{D-93-08}

Thomas Kieninger, Rainer Hoch: Ein Generator mit Anfragesystem für strukturierte Wörterbücher zur Unterstützung von Texterkennung und Textanalyse 125 Seiten

D-93-09

Hans-Ulrich Krieger, Ulrich Schäfer:

TDL ExtraLight User's Guide

35 pages

D-93-10

Elizabeth Hinkelman, Markus Vonerden, Christoph Jung: Natural Language Software Registry

(Second Edition)

174 pages

D-93-11

Knut Hinkelmann, Armin Laux (Eds.):

DFKI Workshop on Knowledge Representation Techniques - Proceedings

88 pages

D-93-12

Harold Boley, Klaus Elsbernd, Michael Herfert, Michael Sintek, Werner Stein:

RELFUN Guide: Programming with Relations and Functions Made Easy 86 pages

D-93-14

Manfred Meyer (Ed.): Constraint Processing Proceedings of the International Workshop at CSAM'93, July 20-21, 1993

264 pages

Note: This document is available only for a nominal charge of 25 DM (or 15 US-\$).

D-93-15

Robert Laux: Untersuchung maschineller

Lernverfahren und heuristischer Methoden im Hinblick auf deren Kombination zur Unterstützung eines Chart-Parsers

86 Seiten

D-93-20

Bernhard Herbig:

Eine homogene Implementierungsebene für einen hybriden Wissensrepräsentationsformalismus 97 Seiten

\section{D-93-21}

Dennis Drollinger:

Intelligentes Backtracking in Inferenzsystemen am Beispiel Terminologischer Logiken 53 Seiten 